\title{
Physical optics and full-wave simulations of transmission of electromagnetic fields through electrically large planar meta-sheets
}

\author{
Ezgi Öziş ${ }^{1}$, Andrey V. Osipov ${ }^{1}$, and Thomas F. Eibert ${ }^{2}$ \\ ${ }^{1}$ Microwaves and Radar Institute, German Aerospace Center, Oberpfaffenhofen, Wessling, 82234, Germany \\ ${ }^{2}$ Chair of High-Frequency Engineering, Technical University of Munich, Munich, 80290, Germany \\ Correspondence to: Ezgi Öziş (ezgi.oezis@dlr.de)
}

Received: 20 December 2016 - Revised: 21 February 2017 - Accepted: 27 February 2017 - Published: 21 September 2017

\begin{abstract}
Ultra-thin metamaterials, called meta-surfaces or meta-sheets, open up new opportunities in designing microwave radomes, including an improved transmission over a broader range of antenna scan angles, tailorable and reconfigurable frequency bands, polarization transformations, one-way transmission and switching ability. The smallness of the unit cells combined with the large electrical size of microwave radomes significantly complicates full-wave numerical simulations as a very fine sampling over an electrically large area is required. Physical optics (PO) can be used to approximately describe transmission through the radome in terms of the homogenized transmission coefficient of the radome wall. This paper presents the results of numerical simulations of electromagnetic transmission through planar meta-sheets (infinite and circularly shaped) obtained by using a full-wave electromagnetic field simulator and a PObased solution.
\end{abstract}

\section{Introduction}

Microwave antennas must be protected against external mechanical (aerodynamic drag, wind) and chemical (ice, rain) effects. Consequently, protective coverings are needed, which are called radomes (radar domes). The shape of the radome must be typically conformal with the shape of the platform (car, aircraft, ship). An ideal microwave radome must be fully transparent at microwave frequencies, at least over the bandwidth of the enclosed antenna. However, losses in the power and distortions in the phase of trans- mitted/received signals caused by realistic radome walls may lead to significant degradation in antenna performance.

The most noticeable effect is the reduction of the transmitted power, which leads to a reduced range of a radar device. A further effect is the growth of the received echo. Reflections, eventually multiple reflections, within the volume enclosed by the radome are the reason for the existence of standing waves in the transmission line of the system, which alters the magnetron frequency ("pulling") and is a reason for the loss of signals. Pulling depends on the magnitude and phase of the standing waves and therefore on the reflection coefficient of the radome wall. Furthermore, refraction in the walls of the radome and phase distortions upon transmission through the radome wall may result in an apparent shift in the angular position of targets (Cady et al., 1948).

If an improved transmission is required at a specific frequency or in a limited frequency band, meta-sheets can be utilized to design the radome walls. Meta-sheets (also metasurfaces, metafilms) are quasi two-dimensional metamaterials that consist of a single layer of electrically small resonators (inclusions) embedded in an electrically thin layer of a dielectric material (substrate). Meta-sheets allow a wide range of possibilities in controlling transmission, reflection and absorption of electromagnetic waves, e.g. Tretyakov (2015). Similarly to metamaterials (Capolino, 2009), metasheets are in general periodic arrays of subwavelength unit cells placed in a substrate. Since the size of the unit cell is much smaller than the wavelength of the incident wave, meta-sheets appear to be effectively homogeneous with the effective material parameters which are much broader than in natural materials and can be adjusted to achieve a desired 
electromagnetic behaviour of the sheet. Meta-sheets can bring new features to microwave radomes such as tailorable and reconfigurable frequency bands, polarization transformations, one-way transmission and switching ability (Öziş et al., 2015, 2016).

Typically, radomes are electrically large structures and, therefore, simulation of transmission and reflection properties of a radome with full-wave numerical methods (FEM, FDTD) can be a difficult, memory- and time-consuming task. An additional complication, specific for meta-sheets and metamaterials, is the need for extremely fine spatial discretization, which results in a very large number of unknowns in the discretized problem. Electromagnetic scattering from electrically large objects may well be calculated by highfrequency approximations based on physical optics (PO), e.g. Albani et al. (2011), Obelleiro-Basteiro et al. (1995), Youssef (1989). The PO equivalent currents induced by an incident wave on a meta-sheet portion of the radome can be calculated from reflection and transmission coefficients of the locally conformal planar infinite meta-sheet (tangent plane approximation). The transmission and reflection coefficients of planar infinite meta-sheets can be relatively easily calculated since the simulation volume can be reduced to a single unit cell of the meta-sheet by using periodicity of the structure. So, PO combined with numerically obtained transmission and reflection coefficients of the tangent meta-sheets can be a good alternative to full-wave methods, but applicability of the results should be validated through comparisons with full-wave simulations.

In this paper, PO is applied to electrically large planar circular meta-sheets to calculate the transmitted electric field. Isotropic ring-shaped metal particles are used as inclusions to make the structure polarization-independent. The high frequency structure simulator (HFSS) (ANSYS, 2016) is used to calculate the effective transmission coefficient of the metasheet (to be employed in the framework of the PO approach) and to perform full-wave simulations of the transmitted field in the presence of an electrically large meta-sheet of finite size. The PO and full-wave electromagnetic field simulation results will be compared on the optical axis of the configuration, where the $\mathrm{PO}$ solution can be expressed in a particularly simple form and the error of the PO solution is expected to be at maximum. The operation frequency is chosen to be at $10 \mathrm{GHz}$.

This paper is organized as follows. In Sect. 2, we briefly review the principles of the $\mathrm{PO}$ approximation and derive a $\mathrm{PO}$ solution for a circular partially transparent plate described by a transmission coefficient. The HFSS setup for full-wave simulations of transmission through an infinite planar metasheet and through a finite electrically large circular metasheet is presented in Sect. 3. In Sect. 4, the PO approach and full-wave electromagnetic field simulation are applied to an electrically large perfectly conducting (PEC) disc to show that the chosen structures are sufficiently large in order that the PO approximation to be valid and then to penetrable

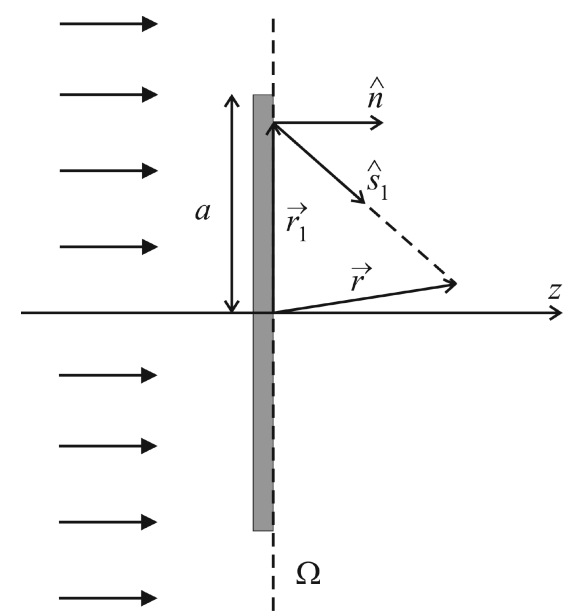

Figure 1. An infinite plane $\Omega$ separates the sources (to the left) and the observation point (to the right). A plane wave is incident perpendicularly to $\Omega$.

meta-sheets of the same diameter. The conclusions are given in Sect. 5. The time dependence $\exp (j \omega t)$ is assumed and omitted throughout.

\section{Theory}

PO approximation is a high-frequency approximation, which in contrast to geometrical optics (GO) accounts for the finite size of the wavelength (Macdonald, 1902; Mentzer, 1955; Osipov and Tretyakov, 2017; Saez de Adana et al., 2011; Ufimtsev, 2014). It can be defined as a combination of GO with the field equivalence principle. According to the equivalence principle the fields scattered by an object can be calculated by integration of equivalent electric and magnetic currents over a surface enclosing the object (Collin, 1960). The equivalent currents are given by tangential electric and magnetic field components on the integration surface. Since the exact fields are a priory unknown, GO approximation for the currents is used, assuming that the integration surface is almost flat on the scale of the wavelength (tangent plane approximation).

\subsection{PO formulation}

Consider an infinite planar semi-transparent screen $\Omega$ at $z=$ 0 , which is described by a transmission coefficient $T(x, y)$. The screen can partially coincide with a side of a physical scatterer but can also be a mathematical surface, in which case $T=1$ (Fig. 1). The screen is illuminated by a linearly polarized plane wave which is incident from the left halfspace normally to the screen:

$\boldsymbol{E}_{\text {inc }}=\boldsymbol{E}_{0} e^{-j k z}$

where $k=\omega \sqrt{\varepsilon_{0} \mu_{0}}$ is the wavenumber, $\omega$ is the angular frequency, $\varepsilon_{0}$ and $\mu_{0}$ are the free-space permittivity and perme- 
ability. At the surface of the screen the transmitted electric field is given by

$\boldsymbol{E}_{\mathrm{tr}}=T(x, y) \boldsymbol{E}_{0}$.

This formula is exact for an infinite planar homogeneous screen with $T(x, y)=$ const. Otherwise, Eq. (2) can be considered as the GO approximation, provided that the transmission coefficient is slowly varying on the scale of the wavelength $\lambda=2 \pi / k$.

To calculate the field at an observation point to the right of the screen in the PO approximation, we use the infinite plane $\Omega$ as the integration surface. The plane separates the field sources (left half-space) and the observation point (right half-space). The equivalent electric and magnetic currents are given by

$\boldsymbol{J}_{\mathrm{m}}=-\hat{n} \times \boldsymbol{E}_{\mathrm{tr}}$,

$\boldsymbol{J}_{\mathrm{e}}=\hat{n} \times \boldsymbol{H}_{\mathrm{tr}}$,

where $\hat{n}$ is the unit vector normal to $\Omega$ and directed into the half-space with the observer, $\boldsymbol{E}_{\text {tr }}$ and $\boldsymbol{H}_{\text {tr }}$ are the electric and magnetic field at the integration surface. The electric field can be expressed through the currents by using the electric and magnetic potentials:

$\boldsymbol{E}_{\mathrm{tr}}=-\frac{1}{\varepsilon_{0}} \nabla \times \boldsymbol{F}-j \omega \boldsymbol{A}+\frac{\nabla(\nabla \cdot \boldsymbol{A})}{j \omega \mu_{0} \varepsilon_{0}}$,

where the potentials are given by the integrals over the surface $\Omega$

$\boldsymbol{F}=\varepsilon_{0} \iint \boldsymbol{J}_{\mathrm{m}}\left(\boldsymbol{r}_{1}\right) g\left(\boldsymbol{r}, \boldsymbol{r}_{1}\right) \mathrm{d} \Omega$,

$\boldsymbol{A}=\mu_{0} \iint \boldsymbol{J}_{\mathrm{e}}\left(\boldsymbol{r}_{1}\right) g\left(\boldsymbol{r}, \boldsymbol{r}_{1}\right) \mathrm{d} \Omega$.

Here,

$g\left(\boldsymbol{r}, \boldsymbol{r}_{1}\right)=\frac{e^{-j k R}}{4 \pi R}$

is the free-space Green function and $R=\left|\boldsymbol{r}-\boldsymbol{r}_{1}\right|$ is the distance between the integration point $\boldsymbol{r}_{1}=\left(x_{1}, y_{1}, 0\right)$ and the observation point $\boldsymbol{r}=(x, y, z)$.

Equations (5), (6) and (7) give an exact representation of the field transmitted through the screen. In the PO approximation, $\boldsymbol{E}_{\text {tr }}$ and $\boldsymbol{H}_{\text {tr }}$ in Eqs. (3) and (4) are assumed to be as in a plane wave, so that their values are determined from formula (2) and from the plane-wave relation $\boldsymbol{H}_{\mathrm{tr}}=Y_{0} \hat{n} \times \boldsymbol{E}_{\text {tr }}$ $\left(Y_{0}=\sqrt{\varepsilon_{0} / \mu_{0}}\right.$ being the free-space admittance of the surrounding medium). Furthermore, for an observation point at a distance greater than a couple of wavelengths from the screen, $k R \gg 1$ and the application of the differential operators in Eq. (5) to the Green function can be approximately evaluated, which gives

$\boldsymbol{E}_{\text {tr }}=\frac{j k}{4 \pi} \iint g\left(\boldsymbol{r}, \boldsymbol{r}_{1}\right)\left(\hat{s}_{1} \times \boldsymbol{J}_{\mathrm{m}}+Z_{0} \hat{s}_{1} \times \hat{s}_{1} \times \boldsymbol{J}_{\mathrm{e}}\right) \mathrm{d} \Omega$, where $Z_{0}=\sqrt{\mu_{0} / \varepsilon_{0}}$ is the intrinsic impedance of the surrounding medium and

$\hat{s}_{1}=\frac{\boldsymbol{r}-\boldsymbol{r}_{1}}{\left|\boldsymbol{r}-\boldsymbol{r}_{1}\right|}$

is the scattering direction as shown in Fig. 1.

In this paper, we assume that a part of the screen is the backside of a homogeneous semi-transparent disc of radius $a$ as shown in Fig. 1, so the transmission coefficient can be written as

$T(x, y)=\left\{\begin{array}{ll}1, & |\rho|>a \\ T_{\text {screen }}, & |\rho| \leq a\end{array}\right.$,

where $\rho$ is the distance from the $z$ axis and $T_{\text {screen }}$ is the transmission coefficient of the disc. Then, for an observation point at the $z$ axis the PO integral (Eq. 9) can be significantly simplified upon introduction of polar coordinates $\left(\rho_{1}, \varphi_{1}\right)$ on $\Omega$ and integration with respect to $\varphi_{1}$, which gives

$$
\begin{gathered}
\boldsymbol{E}_{\mathrm{tr}}^{\mathrm{PO}}(0,0, z)=\boldsymbol{E}_{0} \frac{j k}{4} \int_{0}^{+\infty} e^{-j k \sqrt{\rho_{1}^{2}+z^{2}}} T\left(\rho_{1}\right) \\
\left(2+\frac{2 z}{\sqrt{\rho_{1}^{2}+z^{2}}}-\frac{\rho_{1}^{2}}{\rho_{1}^{2}+z^{2}}\right) \frac{\rho_{1} \mathrm{~d} \rho_{1}}{\sqrt{\rho_{1}^{2}+z^{2}}}
\end{gathered}
$$

The integral can be split up into a contribution from the circular screen and from the rest of the integration surface:

$\boldsymbol{E}_{\mathrm{tr}}^{\mathrm{PO}}=\boldsymbol{E}_{\text {screen }}^{\mathrm{PO}}+\boldsymbol{E}_{\text {rim }}^{\mathrm{PO}}$,

where

$$
\begin{gathered}
\boldsymbol{E}_{\text {screen }}^{\mathrm{PO}}(0,0, z)=T_{\text {screen }} \boldsymbol{E}_{0} \frac{j k}{4} \int_{0}^{a} e^{-j k \sqrt{\rho_{1}^{2}+z^{2}}} \\
\left(2+\frac{2 z}{\sqrt{\rho_{1}^{2}+z^{2}}}-\frac{\rho_{1}^{2}}{\rho_{1}^{2}+z^{2}}\right) \frac{\rho_{1} \mathrm{~d} \rho_{1}}{\sqrt{\rho_{1}^{2}+z^{2}}}
\end{gathered}
$$

and

$$
\begin{gathered}
\boldsymbol{E}_{\mathrm{rim}}^{\mathrm{PO}}(0,0, z)=\boldsymbol{E}_{0} \frac{j k}{4} \int_{a}^{+\infty} e^{-j k \sqrt{\rho_{1}^{2}+z^{2}}} \\
\left(2+\frac{2 z}{\sqrt{\rho_{1}^{2}+z^{2}}}-\frac{\rho_{1}^{2}}{\rho_{1}^{2}+z^{2}}\right) \frac{\rho_{1} \mathrm{~d} \rho_{1}}{\sqrt{\rho_{1}^{2}+z^{2}}} .
\end{gathered}
$$

The term $\boldsymbol{E}_{\text {screen }}^{\mathrm{PO}}$ describes the field transmitted through the disc. In order for the PO method to be applicable, the disc must be electrically large $(k a \gg 1)$, permitting an asymptotic evaluation of the term $\boldsymbol{E}_{\text {rim }}^{\mathrm{PO}}$ by integration by parts. Rewriting Eq. (15) as 


$$
\begin{gathered}
\boldsymbol{E}_{\text {rim }}^{\mathrm{PO}}(0,0, z)=-\boldsymbol{E}_{0} \frac{1}{4} \int_{a}^{+\infty}\left(2+\frac{2 z}{\sqrt{\rho_{1}^{2}+z^{2}}}-\frac{\rho_{1}^{2}}{\rho_{1}^{2}+z^{2}}\right) \\
\frac{\partial}{\partial \rho_{1}} e^{-j k \sqrt{\rho_{1}^{2}+z^{2}}} \mathrm{~d} \rho_{1},
\end{gathered}
$$

integrating by parts once and neglecting the asymptotically smaller terms, one obtains the formula

$$
\begin{aligned}
& \boldsymbol{E}_{\text {rim }}^{\mathrm{PO}}(0,0, z) \approx \boldsymbol{E}_{0} \frac{1}{4}\left(2+\frac{2 z}{\sqrt{a^{2}+z^{2}}}-\frac{a^{2}}{a^{2}+z^{2}}\right) \\
& e^{-j k \sqrt{a^{2}+z^{2}}}
\end{aligned}
$$

which can be interpreted as a contribution from the rim of the disc. Equations (13), (14) and (17) describe the PO solution for the electric field transmitted through the homogeneous semi-transparent circular disc.

\section{Simulation of meta-sheets and unit cells in HFSS}

As full-wave electromagnetic field simulator, we use HFSS which is based on the finite element method (Huebner et al., 2001). The simulator divides the solution domain into a finite number of elements. After converting the entire domain into a discretised domain, a suitable interpolation function is chosen. The interpolation functions can be first, second, or higher order polynomials. The unknown fields are approximated by known basis functions with unknown expansion coefficients. The expansion coefficients are determined by solving a system of linear equations.

\subsection{HFSS simulation of infinite planar meta-sheets}

Simulation of infinite planar meta-sheets consisting of identical unit cells can be reduced to a single unit cell with boundary conditions accounting for periodicity of the structure. In the framework of HFSS, a unit cell as shown in Fig. 2 is placed in an air-box with master/slave boundary conditions and Floquet ports for excitation (Fig. 3). The surfaces of the air-box which are perpendicular to the $x$ and $y$ axes are chosen to be Master/slave boundaries. The master and slave boundaries are paired, and their surfaces should have identical size and shape. The meta-sheet consists of copper rings placed on a dielectric substrate (FR4 epoxy). The thickness of the rings is $18 \mu \mathrm{m}$. A linearly polarized plane wave is incident normally to the meta-sheet, and the operation frequency is $10 \mathrm{GHz}$. The HFSS simulation volume is an air-box of the length $60 \mathrm{~mm}$ with a unit cell inside. Two designs with different size of the rings and of the unit cells are studied, as shown in Table 1. The thickness of the substrate, which is $2 \mathrm{~mm}$, is the same for both designs. The calculated values of the magnitude and phase of the transmission coefficient can be found in Table 1. These values are used in the framework

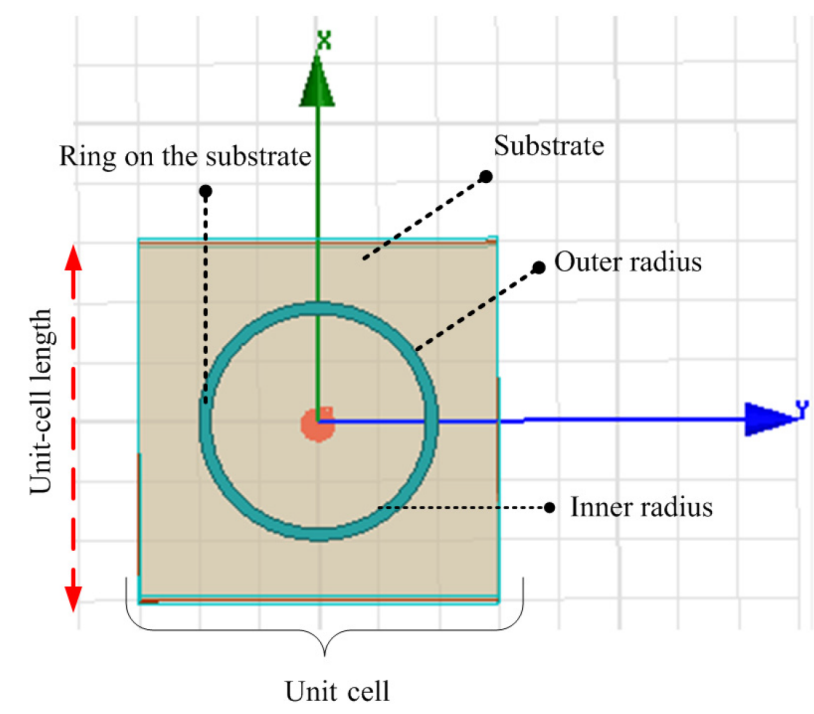

Figure 2. The unit cell of a meta-sheet consists of a copper ring embedded in a square piece of a dielectric substrate (FR4 epoxy).

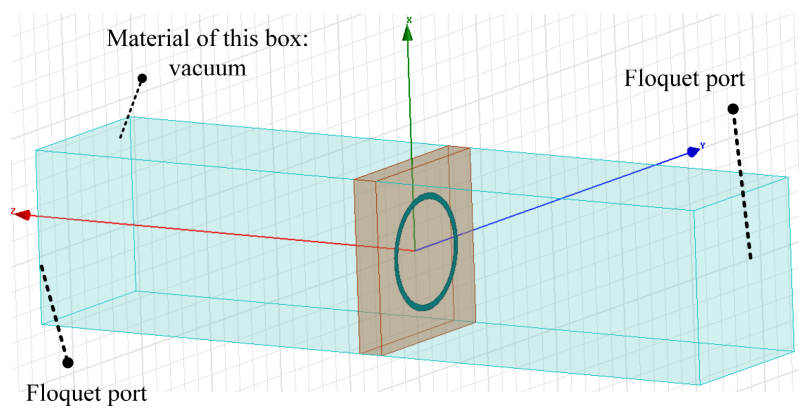

Figure 3. HFSS simulation volume.

of PO calculations of transmission through finite structures in Sect. 4.

Table 1. The parameters of the unit cells and the corresponding transmission coefficients obtained with HFSS. The outer radius and inner radius describe the shape of the rings.

\begin{tabular}{llrrr}
\hline $\begin{array}{l}\text { Outer } \\
\text { radius }\end{array}$ & $\begin{array}{l}\text { Inner } \\
\text { radius }\end{array}$ & $\begin{array}{r}\text { Unit-cell } \\
\text { length }\end{array}$ & $\begin{array}{r}\text { Phase of } \\
\text { transmission } \\
\text { coefficient }\end{array}$ & $\begin{array}{l}\text { Transmission } \\
\text { coefficient }\end{array}$ \\
\hline $1.6 \mathrm{~mm}$ & $1.44 \mathrm{~mm}$ & $4.8 \mathrm{~mm}$ & $-42.3^{\circ}$ & $-2.37 \mathrm{~dB}$ \\
$4.6 \mathrm{~mm}$ & $4.14 \mathrm{~mm}$ & $13.8 \mathrm{~mm}$ & $16.3^{\circ}$ & $-1.55 \mathrm{~dB}$ \\
\hline
\end{tabular}

\subsection{HFSS simulation of meta-sheets of finite extent}

We have studied transmission through circular planar metasheets 160 and $340 \mathrm{~mm}$ in diameter (Fig. 4). At the operation frequency, the discs are about $5 \lambda$ and $11 \lambda$ and therefore electrically large. The PEC discs of the same size as the metasheets (Fig. 5) have been studied first to see whether the discs 


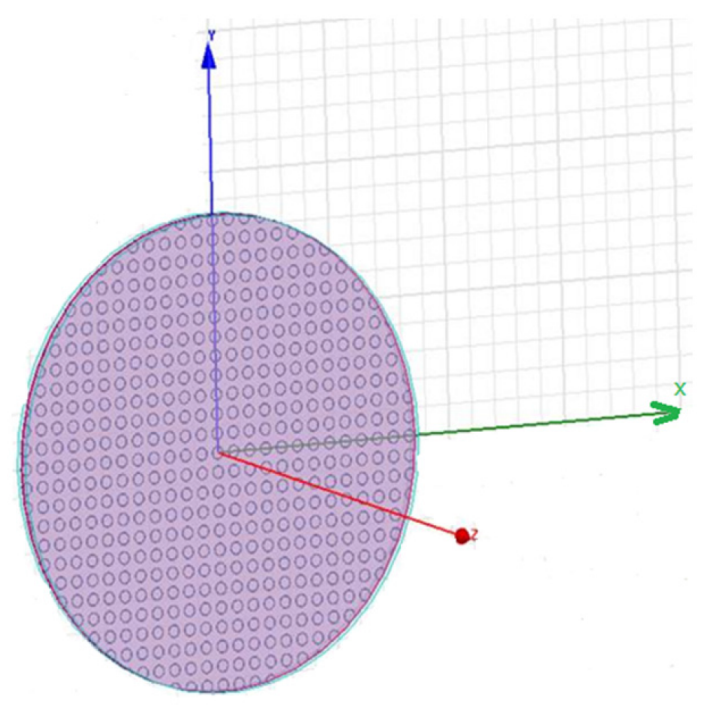

Figure 4. The circular meta-sheet in HFSS: electrically small rings are embedded into an electrically large circular FR4 disc.

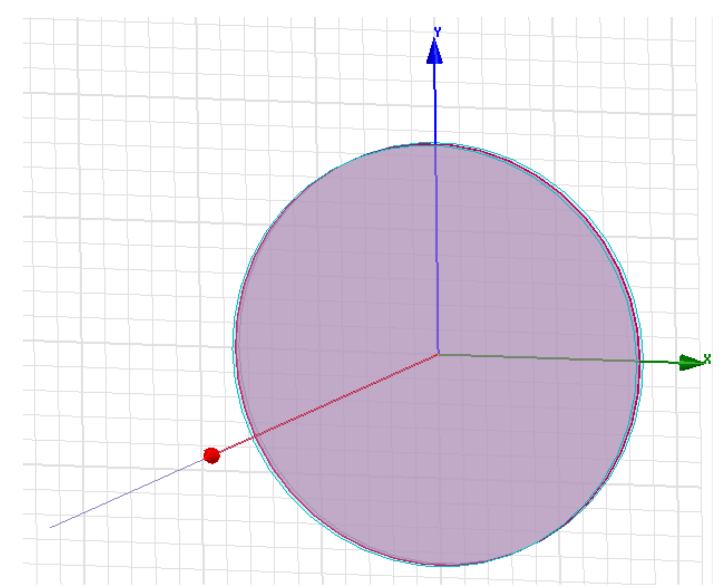

Figure 5. The electrically large circular PEC disc is of the same size as the meta-sheet in Fig. 4.

are large enough in order for the PO approximation to apply. The meta-sheet parameters are the same as in Sect. 3.1. HFSS is used to calculate the scattered field (total field minus incident field) behind the discs. The discs are placed in a cylindrical air-box, and to make the fields compliant with the radiation condition, a hybrid boundary condition is applied at the whole boundary of the cylindrical air-box (ANSYS, 2012). This form of the boundary condition is a computationally efficient way of accounting for radiation in electrically large problems. The scattered electric fields along the radiation line are then determined with the combination of the finite element and boundary integral equations methods. The excitation is a plane wave incident normally on the discs from the left. The field is sampled on the $z$ axis between 10 and $1000 \mathrm{~mm}$ on the right of the discs.

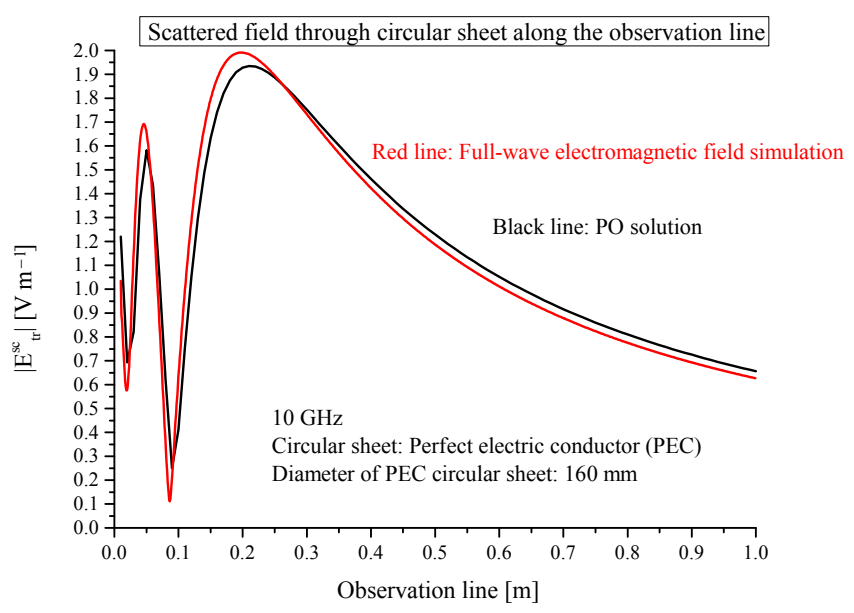

Figure 6. Scattered electric field along the observation line. The diameter of the circular PEC disc is $160 \mathrm{~mm}$.

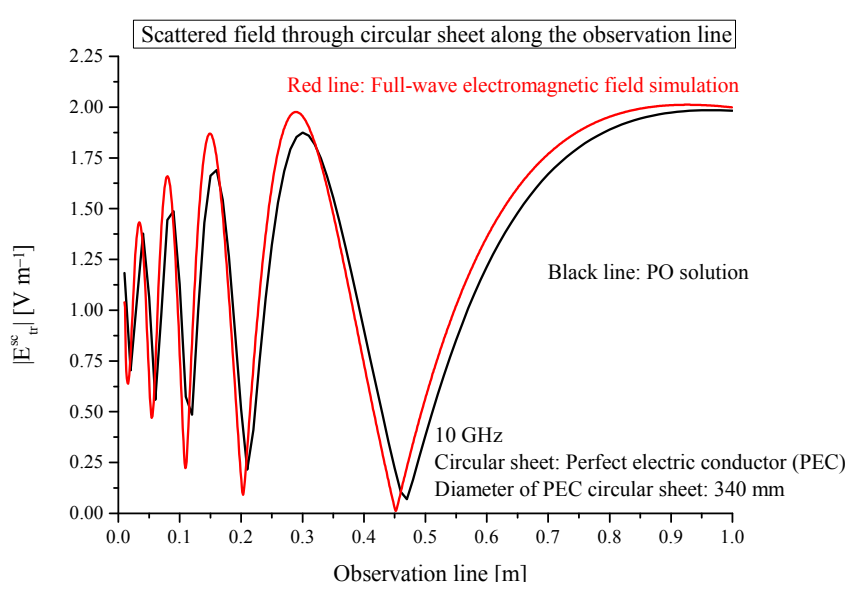

Figure 7. The same as in Fig. 6 but for a PEC disc with the diameter $340 \mathrm{~mm}$.

\subsection{Comparison of results of $\mathrm{PO}$ approach and full-wave electromagnetic field simulation}

The PO solution given by Eqs. (13), (14) and (17) has been applied to calculating the field scattered by the circular discs (PEC and meta-sheet) and compared with the results of the full-wave simulations. The integral in the PO solution (14) has been calculated by numerical integration using an adaptive integration algorithm, which recursively subdivides the integration region as needed.

\subsection{Electrically large PEC disc}

The results of simulations for the PEC discs with the diameter 160 and $340 \mathrm{~mm}$ are shown in Figs. 6 and 7. The agreement between the PO solution and the full-wave simulation is very good, which suggests that the size of the discs is sufficiently large in order for the PO solution to apply. 


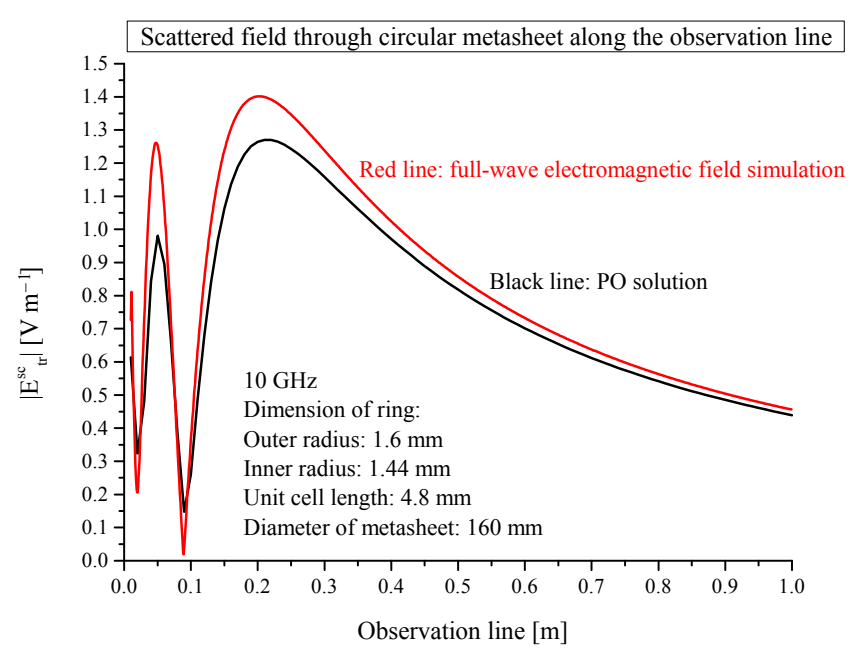

Figure 8. Scattered electric field along the observation line. The diameter of electrically large meta-sheet is $160 \mathrm{~mm}$.

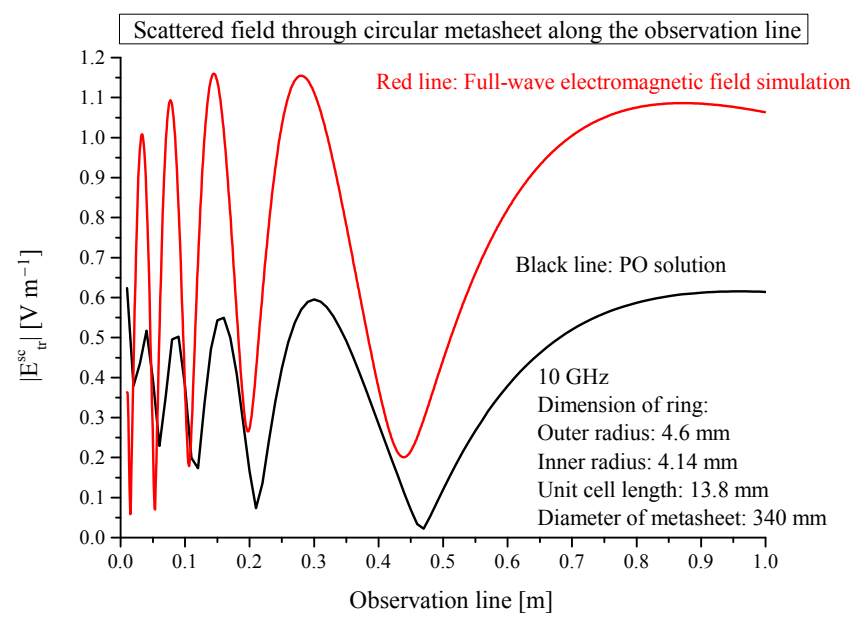

Figure 9. Scattered electric field along the observation line. The diameter of electrically large meta-sheet is $340 \mathrm{~mm}$.

\subsection{Electrically large meta-sheet discs}

Meta-sheets approach homogeneous materials as the size of the unit cells gets significantly smaller than the wavelength $(\lambda / 5$ and smaller) (Ramakrishna and Grzegorczyk, 2008). With unit cells larger than a half wavelength, the structure can no longer be considered homogeneous as it starts to act as a diffraction array producing side lobes in the transmitted and reflected fields. Because of that, the unit cell has to be kept significantly smaller than the wavelength, implying a very large number of unit cells on an electrically large metasheet. For the disc of $160 \mathrm{~mm}$ in diameter the unit cells length is $4.8 \mathrm{~mm}$, which is about $\lambda / 6$, and the structure is effectively homogeneous. A comparison between PO and full-wave solution in Fig. 8 shows a good agreement, though the error is slightly greater than in the PEC case (see Fig. 6). The PO solution correctly predicts the position of maxima and minima, and it is expected that the accuracy will improve with growing electrical size of the disc.

For the disc of $340 \mathrm{~mm}$ in diameter, larger unit cells $(13.8 \mathrm{~mm})$ have been taken in order to keep the discretised problem numerically tractable. However, such unit cells are about $\lambda / 2$ in size, and the material cannot be regarded as effectively homogeneous. This explains the significant disagreement between the PO and full-wave solutions in Fig. 9.

\section{Conclusion}

For calculation of fields transmitted through finite electrically large meta-sheet structures, a hybrid method using PO approximation with transmission coefficients obtained from full-wave calculation for an infinite planar periodic structure has been proposed.

A comparison of the results of full-wave numerical simulations with the PO predictions for finite planar structures of moderately large electrical size has been performed. The results are in a good agreement as long as, on the one hand, the size of the structure is greater than several wavelengths and, on the other hand, the unit cells are sufficiently small in order to make the sheet effectively homogeneous. As soon as one or both of these conditions are not fulfilled, the PO approach does not apply any more.

Full-wave numerical calculations of meta-sheets are limited to structures of several wavelengths in size. Application to larger meta-sheets is hardly possible because of difficult meshing, large memory consumption and too long computation times. The PO approach discussed in this paper is a good alternative applicable to electrically large structures, planar or curved, provided that the meta-sheet is effectively homogeneous.

Data availability. The underlaying results of numerical simulations are available from the authors.

Competing interests. The authors declare that they have no conflict of interest.

The article processing charges for this open-access publication were covered by a Research Centre of the Helmholtz Association.

Edited by: R. Schuhmann

Reviewed by: two anonymous referees 


\section{References}

Albani, M., Carluccio, G., and Pathak, P. H.: Uniform ray description for the PO scattering by vertices in curved surface with curvilinear edges and relatively general boundary conditions, IEEE T. Antenn. Propag., 59, 1587-1596, https://doi.org/10.1109/TAP.2011.2123062, 2011.

ANSYS: HFSS for electrically large antenna system design: Hybrid Simulation Technology, ANSYS, Inc., available at: http://www.designspace.com/staticassets/ (last access: November 2016), 2012.

ANSYS: ANSYS HFSS features, ANSYS, Inc., available at: http://www.ansys.com/products/electronics/ansys-hfss/ hfss-features, last access: November 2016.

Cady, W. M., Karelitz, M. B., Turner, L. A., and Herb, R. G.: Radar Scanners and Radomes, Massachusetts Institute of Technology Radiation Laboratory Series, 26, McGraw-Hill, New York, Toronto, London, 1948.

Capolino, F.: Metamaterials Handbook: Theory and Phenomena of Metamaterials, CRC Press, Boca Raton, USA, 2009.

Collin, R. E.: Field Theory of Guided Waves, McGraw-Hill, New York, Toronto, London, 1960.

Huebner, K. H., Dewhirst, L. D., Smith, D. E., and Byrom, T. G.: The Finite Element Method for Engineers, Wiley, New York, Chichester, Weinheim, Brisbane, Singapore, Toronto, 2001.

Macdonald, H. M.: Electric Waves, Cambridge University Press, Cambridge, UK, 1902.

Mentzer, J. R.: Scattering and Diffraction of Radio Wave, Pergamon Press, London, New York, 1955.
Obelleiro-Basteiro, F., Luis Rodriguez, J., and Burkholder, R. J.: An iterative physical optics approach for analyzing the electromagnetic scattering by large open-ended cavities, IEEE T. Antenn. Propag., 43, 356-361, https://doi.org/10.1109/8.376032, 1995.

Osipov, A. V. and Tretyakov, S. A.: Modern Electromagnetic Scattering Theory with Applications, Wiley, Chichester, UK, 2017.

Öziş, E., Osipov, A. V., and Eibert, T. F.: Enhancing microwave radomes with metamaterials, Kleinheubacher Tagung, 28-30 September 2015, Miltenberg, Germany, KHT2015-B-10, 2015.

Öziş, E., Osipov, A. V., and Eibert, T. F.: Physical optics and full wave simulations of transmission of electromagnetic fields through electrically large planar meta-sheets, Kleinheubacher Tagung, 26-28 September 2016, Miltenberg, Germany, KH2016B-18, 2016.

Ramakrishna, S. A. and Grzegorczyk, T. M.: Physics and Applications of Negative Refractive Index Materials, CRC Press, Boca Raton, USA, 2008.

Saez de Adana, F., Gutiérrez, O., Gonzáles, I., Cátedra, M. F., and Lozano, L.: Practical Applications of Asymptotic Techniques in Electromagnetics, Artech House, Norwood, USA, 2011.

Tretyakov, S. A.: Metasurfaces for general transformations of electromagnetic fields, Philos. T. R. Soc. A, 373, 20140362, https://doi.org/10.1098/rsta.2014.0362, 2015.

Ufimtsev, P. Y.: Fundamentals of the Physical Theory of Diffraction, Wiley, Hoboken, USA, 2014.

Youssef, N. N.: Radar cross section of complex target, P. IEEE, 77, 722-734, https://doi.org/10.1109/5.32062, 1989. 